\title{
Veno-Arterial Extracorporeal Membrane Oxygenation for Severe Heatstroke with Refractory Hemodynamic Failure
}

\author{
Masahide Fujita Keita Miyazaki Momoko Horiguchi Kouji Yamamoto \\ Shingo Ito Hidetada Fukushima \\ Department of Emergency and Critical Care Medicine, Nara Medical University, Kashihara \\ City, Japan
}

\begin{abstract}
A 68-year-old male was found unconscious in a hot bathtub on a cold winter's day and was brought to our hospital. On arrival, the patient's systolic blood pressure was $65 \mathrm{~mm} \mathrm{hg}$, heart rate was 134 beats per min, and rectal temperature was $40.5^{\circ} \mathrm{C}$. A large volume infusion of acetated Ringer's solution and vasopressors were administered as an immediate treatment for circulatory failure due to heatstroke. Although the circulatory failure temporarily improved, the blood pressure dropped almost to the point of cardiac arrest. Veno-arterial extracorporeal membrane oxygenation (VA-ECMO) was implemented and the hemodynamics were soon stabilized. Subsequently, VA-ECMO was withdrawn on the third hospital day. The patient recovered enough to feed normally on the 40th hospital day and was transferred to another hospital on the 116th hospital day. Based on this case study, we consider that VA-ECMO implementation before cardiac arrest might be a suitable strategy.
\end{abstract}

\section{Introduction}

Heatstroke is generally defined as an "impairment of physical adaptation under hot environment [1]." The number of patients suffering from heatstroke is increasing every year as a result of global warming [2]. Heatstroke is generally more common in the summer months, but it can occur even in the winter if the temperatures rise to abnormally high levels [3]. Severe heatstroke can result in central nervous system disorders, liver and renal dysfunction, and/or coagulopathy. Furthermore, patients can develop severe hemodynamic failure that requires some form of circulatory support. Here, we report an elderly case of severe 
Table 1. Laboratory data on admission

\begin{tabular}{llll}
\hline Peripheral blood & & Biochemistry & \\
WBC & $3,300 / \mathrm{mm}^{3}$ & CRP & $0.12 \mathrm{mg} / \mathrm{dL}$ \\
$\mathrm{Hb}$ & $12.8 \mathrm{~g} / \mathrm{dL}$ & Alb & $3.5 \mathrm{~g} / \mathrm{dL}$ \\
$\mathrm{Hct}$ & $37.7 \%$ & AST & $86 \mathrm{U} / \mathrm{L}$ \\
$\mathrm{Plt}$ & $18.3 \times 10^{4} / \mathrm{mm}^{3}$ & ALT & $17 \mathrm{U} / \mathrm{L}$ \\
Coagulation test & & LDH & $335 \mathrm{U} / \mathrm{L}$ \\
PT & $64 \%$ & CK & $237 \mathrm{U} / \mathrm{L}$ \\
APTT & $<23 \mathrm{~s}$ & BUN & $12 \mathrm{mg} / \mathrm{dL}$ \\
FDP & $17.3 \mu \mathrm{g} / \mathrm{mL}$ & Cre & $1.21 \mathrm{mg} / \mathrm{dL}$ \\
D-dimer & $8.4 \mu \mathrm{g} / \mathrm{mL}$ & T-Bil & $0.6 \mathrm{mg} / \mathrm{dL}$ \\
\hline
\end{tabular}

heatstroke with refractory hemodynamic failure that responded to veno-arterial extracorporeal membrane oxygenation (VA-ECMO).

\section{Case Presentation}

A 68-year-old male was found unconscious in a bathtub, immersed in hot water up to his chest on a cold winter day. The patient had been in the bath for a number of hours before being found and brought to our hospital. The patient had a history of alcoholic liver disease, hypertension, type 2 diabetes, and hyperlipidemia. On arrival, his vital signs were as follows: GCS E1V1M2; core body temperature (bladder), $40.5^{\circ} \mathrm{C}$; systolic blood pressure, $65 \mathrm{~mm}$ h; heart rate, 134 beats per min; respiratory rate, 31 breaths per min; and saturation on pulse oximetry, $100 \%$ under $10 \mathrm{~L} / \mathrm{min}$ of oxygen supply. His body surface was red in color. His initial laboratory findings showed mild renal dysfunction and mild coagulopathy (Table 1). A chest and abdominal CT scan and brain MRI revealed no abnormalities.

The patient was diagnosed with severe heatstroke; as such, body surface cooling with an ice bag, intragastric infusion of cold water, and cold fluid administration were started. The patient's core temperature dropped to $37^{\circ} \mathrm{C}$ after $1 \mathrm{~h}$ of treatment. Administration of a large volume infusion of crystalloid solution and a continuous infusion of $0.1 \mathrm{mg} / \mathrm{kg} / \mathrm{min}$ of noradrenaline for hypotension improved the systolic blood pressure to $121 \mathrm{~mm} \mathrm{hg}$. The patient was then transferred to the intensive care unit.

After intensive care unit admission, the patient's hemodynamics deteriorated. Echocardiography at the bedside showed no apparent asynergy or pericardial effusion collection. The dose of noradrenaline was increased to $0.3 \mu \mathrm{g} / \mathrm{kg} / \mathrm{min}$, and a large volume infusion load was continued. The patient remained unresponsive and the blood pressure dropped to a level where the carotid artery was barely palpable. Up to this point, the total amount of infusion was 7,500 $\mathrm{mL}$ for $6 \mathrm{~h}$ after hospital arrival and the radial artery systolic blood pressure was $30 \mathrm{~mm}$ hg. We determined that the patient needed hemodynamic support with VA-ECMO. VA-ECMO was implemented as follows: right femoral vein removal - left femoral artery transmission, condition: $3,590 \mathrm{rpm}, \mathrm{FiO}_{2}$ 1.0, Flow $3.7 \mathrm{~L} / \mathrm{min}$. We also initiated blood transfusion to maintain circulatory volume. In contrast, we maintained the dose of vasopressor since the color of the peripheral limbs was pale due to circulatory insufficiency and the high dose of noradrenaline. We added hydrocortisone instead, taking into account the possible adrenal insufficiency. The patient stabilized after VA-ECMO and the hemodynamics markedly improved. VA-ECMO was withdrawn on the 3rd hospital day (Fig. 1). Despite complications, 
Fig. 1. Clinical course. VA-ECMO was introduced $6 \mathrm{~h}$ after admission and successfully withdrawn on 3rd hospital day. VA-ECMO, veno-arterial extracorporeal membrane oxygenation.

Fujita et al.: VA-ECMO for Severe Heatstroke with Refractory Hemodynamic Failure <!--Q1: Please check whether the right running head is correct; else provide another one of not more than 80 characters, including spaces.-->

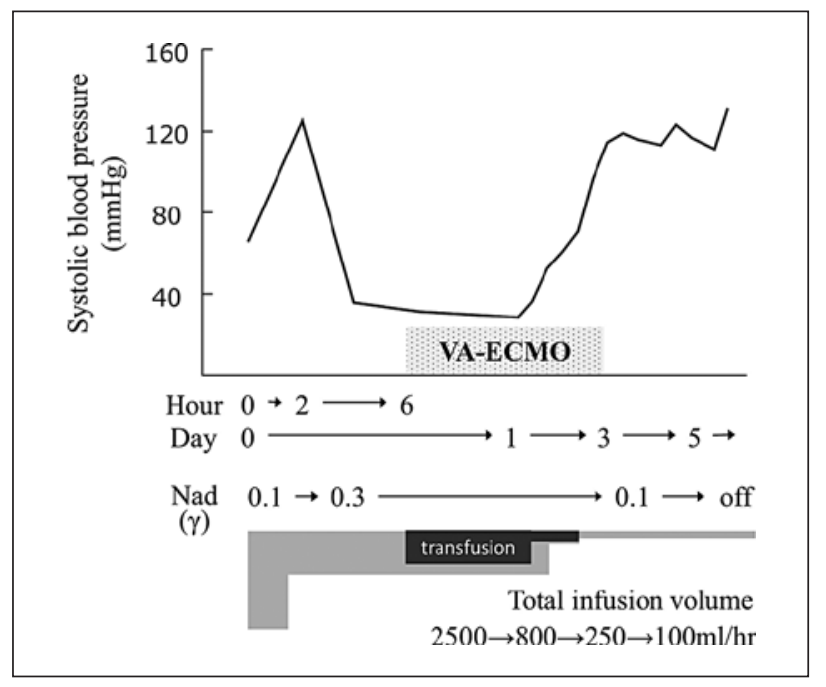

including a rectal ulcer with melena, liver dysfunction, renal dysfunction, disseminated intravascular, and left lower limb ischemia, - the patient recovered to oral intake and was transferred to another hospital on the 116th hospital day.

\section{Discussion}

This patient experienced fatal circulatory failure due to heatstroke. VA-ECMO successfully improved the deteriorated circulatory status and the patient was discharged without neurological sequela. After performing an extensive literature search, we could not find reports of using VA-ECMO for severe circulatory failure due to heatstroke. We thus believe that this is the 1st case for successful VA-ECMO-based treatment of this condition.

Heatstroke normally occurs during the summer season; however, it is common in Japan for the elderly to bathe in very hot water during the winter months. Exposure to such hot water might mimic the conditions causative of heatstroke. Some reports have indicated that body temperatures can accidentally reach up to $40^{\circ} \mathrm{C}$ as a result of bathing in hot water [4]. In the present case, we presumed that the patient lost consciousness and thus could not get out of the hot bathtub: the prolonged period spent in the hot water led to heatstroke.

Severe heatstroke causes hypovolemic shock due to severe dehydration, as well as sepsislike distributive shock due to hypercytokinemia from vascular endothelial dysfunction [5]. Despite understanding the nature of these clinical conditions, the mechanism of circulatory failure is unclear [6]. Consequently, management for severe circulatory failure is not established. Circulatory support by VA-ECMO has been recommended if necessary [1], but there is no clear evidence for its efficacy. We identified a report describing VA-ECMO implementation for refractory shock from sepsis. Here, Park et al. [7] evaluated 32 cases who underwent VA-ECMO for septic shock and concluded that cardiac arrest before VA-ECMO was significantly associated with a lower risk of in-hospital mortality [7]. In our case, we presumed that the patient's initial circulatory failure improved after fluid resuscitation and that another pathological mechanism such as distributive shock might have contributed to the recurrent circulatory failure. The patient's circulatory status deteriorated rapidly, close to the point of a cardiac arrest. The use of other vasopressors was not possible because the decrease in blood pressure to $30 \mathrm{~mm}$ hg was very rapid. Therefore, we decided to implement VA-ECMO, and the patient was successfully resuscitated. As proposed by Park et al. [7], we speculate that 
VA-ECMO before cardiac arrest was the key to survival in this case. This case report indicates that VA-ECMO should be considered as a circulatory management option for severe heatstroke with refractory hypotension or collapse in a short period of time even after conventional management. Further studies are now warranted to determine the clinical indications for and effectiveness of VA-ECMO in such cases.

\section{Conclusions}

Heatstroke is a life-threatening condition that can cause severe circulatory failure and even cardiac arrest. In this case, VA-ECMO promoted recovery and prevented cardiac arrest. Further studies are now required to establish the indications for VA-ECMO in the context of severe heatstroke.

\section{Statement of Ethics}

Our institution does not require ethical approval for reporting individual cases or case series. Written informed consent was obtained from the patient for anonymized information to be published in this article.

\section{Conflict of Interest Statement}

The author(s) declared no potential conflicts of interest with respect to the research, authorship, and/or publication of this article.

\section{Funding Source}

The author(s) received no financial support for the research, authorship, and/or publication of this article.

\section{References}

1 Epstein Y, Yanovich R. Heatstroke. N Engl J Med. 2019;380(25):2449-59.

2 Murakami S, Miyatake N, Sakano N. Changes in air temperature and its relation to ambulance transports due to heat stroke in all 47 prefectures of Japan. J Prev Med Public Health. 2012;45(5):309-15.

3 Zhuang Y, Dai LF, Diao RZ. Multiple organ dysfunction due to heatstroke after sauna: case report and review of the literature. JRSM Open. 2017;8(7):2054270417702313.

4 Suzuki M, Shimbo T, Ikaga T, Hori S. Incidence and characteristics of bath-related accidents. Intern Med. 2019; 58(1):53-62.

5 Miyake Y. Pathophysiology of heat illness: thermoregulation, risk factors, and indicators of aggravation. Japan Med Assoc J. 2013;56(3):167-73.

6 Yeo TP. Heat stroke: a comprehensive review. AACN Clin Issues. 2004;15(2):280-93.

7 Park TK, Yang JH, Jeon K, Choi SH, Choi JH, Gwon HC, et al. Extracorporeal membrane oxygenation for refractory septic shock in adults. Eur J Cardiothorac Surg. 2015;47(2):e68-74. 